\title{
POLITIQUE INDUSTRIELLE ET GOUVERNANCE : L'EXPÉRIENCE DES CLUSTERS INNOVANTS EN ESPAGNE
}

Josep-antoni Ybarra et Rafael Doménech Sanchez

De Boeck Supérieur | Innovations

$2014 / 2-n^{\circ} 44$
pages 105 à 126

ISSN 1267-4982

Article disponible en ligne à l'adresse:

http://www.cairn.info/revue-innovations-2014-2-page-105.htm

Pour citer cet article :

Ybarra Josep-antoni et Sanchez Rafael Doménech, « Politique industrielle et gouvernance : l'expérience des clusters innovants en Espagne »,

Innovations, $2014 / 2 \mathrm{n}^{\circ} 44$, p. 105-126. DOI : 10.3917/inno.044.0079

Distribution électronique Cairn.info pour De Boeck Supérieur.

(c) De Boeck Supérieur. Tous droits réservés pour tous pays.

La reproduction ou représentation de cet article, notamment par photocopie, n'est autorisée que dans les limites des conditions générales d'utilisation du site ou, le cas échéant, des conditions générales de la licence souscrite par votre établissement. Toute autre reproduction ou représentation, en tout ou partie, sous quelque forme et de quelque manière que ce soit, est interdite sauf accord préalable et écrit de l'éditeur, en dehors des cas prévus par la législation en vigueur en France. II est précisé que son stockage dans une base de données est également interdit. 


\title{
POLITIQUE INDUSTRIELLE ET GOUVERNANCE : L'EXPÉRIENCE DES CLUSTERS INNOVANTS EN ESPAGNE*
}

\author{
Josep-Antoni YBARRA \\ Université d'Alicante, Espagne \\ ybarra@ua.es \\ Rafael Doménech SANCHEZ \\ Université Miguel Hernández-Elche, Espagne \\ r.domenech@umh.es
}

En août 2006, le gouvernement espagnol a publié un arrêté ministériel dans lequel il jetait les bases pour développer une politique industrielle de soutien aux groupements d'entreprises ; son objectif était de promouvoir des groupements d'entreprises - en obtenant une masse suffisante - en vue de susciter une dynamique d'innovation à caractère territorial et sectoriel. Le présent travail fait une première évaluation de la signification de cette politique menée entre 2007 - première année pendant laquelle ces montants prévus au programme peuvent s'appliquer - et à la fin 2011 - la dernière année pour laquelle des données sont disponibles. Pour cela, nous analysons les critères généraux pour la génération des Groupements d'Entreprises Innovantes (GEI) leurs liens avec leur environnement productif local, ainsi que les aspects de la gouvernance relatifs à la constitution des GEI.

\section{POLITIQUES INDUSTRIELLES POUR LES SYSTĖMES PRODUCTIFS LOCAUX : LES GROUPEMENTS D'ENTREPRISES INNOVANTES EN ESPAGNE}

Jusqu'à la fin des années quatre-vingt, la politique industrielle était axée sur les mesures et les programmes visant à l'impulsion et/ou la réorientation

\footnotetext{
* Les auteurs apprécient les commentaires et observations formulés par les évaluateurs sur une version précédente. Sans aucun doute, cela a contribué à l'amélioration du texte final. Le texte demeure sous la seule responsabilité de leurs auteurs.
} 
des activités de production en crise à travers l'aide directe aux entreprises et aux activités (Cowling et al., 1999 ; Bianchi, Labory, 2006). Cette stratégie de politique industrielle a changé considérablement lorsque les initiatives se sont focalisées sur le territoire comme porteur des activités et des entreprises. On voit ainsi apparaître une ligne de travail autour du groupe GREMI ${ }^{1}$ qui met l'accent sur les politiques industrielles de deuxième et de troisième génération (Maillat et al., 1993) qui soulignent l'importance de la mise en valeur des conditions endogènes du territoire et, ensuite, la capacité de créer un avantage comparatif dans un environnement de plus en plus mondialisé. Après la crise du fordisme et l'importance croissante des petites et moyennes entreprises, l'élément territorial devient le centre d'attention des chercheurs et des responsables politiques (Piore, Sabel, 1984). Dans le nouveau paradigme pour l'action, les principaux acteurs des politiques industrielles ne sont plus les individus, ni les activités ou les entreprises d'un groupe, mais l'espace-lieu que ces derniers occupent. Sur la base des travaux de Becattini (1979), Brusco (1982), ou Dei Ottati (1994), des concepts classiques, tels que le district industriel (Marshall, 1920) ont repris vie. Ầ la différence de la définition néoclassique traditionnelle, basée sur les économies externes et d'agglomération territoriale et la spécialisation, la théorie du district met l'accent sur l'interdépendance entre les entreprises, la dichotomie de la concurrence et la coopération, et l'importance des systèmes culturels et des traditions sociales (Dei Ottati 1994). Parallèlement, le travail de Porter sur les clusters des systèmes productifs a joué un rôle sur la reconnaissance par les responsables politiques des avantages offerts par la proximité territoriale. D'après les termes utilisés à l'origine par Porter (1998), un cluster est un groupe géographiquement proche d'entreprises reliées entre elles par des éléments communs et complémentaires. Les relations concurrence-coopération qui s'établissent au sein des groupements offrent des bénéfices économiques pour les entreprises de l'agglomération, comme résultat d'une productivité et une efficience accrues, d'un développement de la capacité d'innovation et d'un taux plus élevé de création de nouvelles entreprises.

En définitive, la dimension territoriale de la structure sociale et institutionnelle devient un élément de base dans l'explication des différences de performance des systèmes productifs locaux (Asheim, 2003). L'étude des relations entre l'environnement institutionnel, l'activité innovatrice et les résultats des régions a été abordée dans un nombre croissant de travaux dans une approche plus large des Systèmes Régionaux d'Innovation (Cooke, 1992). Suivant cette approche, le processus interactif d'apprentissage qui se produit dans un cadre local mène à la création et à l'absorption de connaissances basées sur

1. GREMI : Groupe de Recherche Européen sur les Milieux Innovateurs. 
des ressources uniques et spécifiques que l'on ne trouve pas ailleurs et qui, par conséquent, sont difficilement transférables (Maskell, Malmberg, 1999). La proximité territoriale favorise la collaboration entre des institutions appartenant à des domaines différents dans la transmission de connaissances tacites et difficilement codifiables (Ponds, Van Oort, Frenken, 2007). Dans le modèle traditionnel, la connaissance était générée et cumulée dans les universités et les établissements publics de recherche répondant aux intérêts académiques. Dans les économies post-industrielles, elle devient une connaissance socialement répartie axée sur l'identification et la solution de problèmes pratiques qui se posent au quotidien (Gibbons et al., 1994 ; Foray, 2004). Dans le nouveau mode de production de connaissances, les interactions entre les principaux acteurs institutionnels du système d'innovation - l'État, l'Université et l'entreprise - ne répondent plus à une dynamique linéaire et prévisible (Etzkowitz, Leydesdorff, 2000) mais elles se trouvent dans une transition permanente où la communication et la négociation entre chacune des parties - le gouvernement, l'académie et l'industrie - est cruciale pour favoriser la co-évolution du système d'innovation, faisant jouer aux politiques publiques un rôle fondamental dans le renforcement et le contrôle de cette dynamique.

En conclusion, les différences dans la dotation institutionnelle des régions permettraient d'expliquer les variations dans la performance et dans l'obtention d'avantages compétitifs permanents dans un environnement mondialisé. Cette constatation est peut-être l'argument majeur en faveur de la régionalisation de la politique d'innovation et la base sur laquelle s'est appuyée la diffusion croissante des «nouvelles politiques régionales » dirigées vers les réseaux d'entreprises, les clusters productifs et la création de compétences locales. Mais, parallèlement à leur application croissante, l'expérience a mis en évidence la difficulté de connaître de manière empirique leurs effets finaux sur le développement régional en se basant sur les méthodes et les instruments traditionnels d'évaluation (Diez, 2001 ; Borras, Tsagdis, 2008; Martin, Sunley 2003 ; Asheim, 2003 ; Doloreux, Parto 2005 ; Visser, Atzema 2008 ; Hospers et al., 2008). Ainsi, au cours des dernières années, de nombreux travaux ont révisé de manière critique les expériences d'évaluation des résultats de ces politiques (Fromhold-Eisebith, Eisebith 2008) en proposant des approches alternatives aux modèles économétriques, à l'analyse d'impact ou à l'analyse coût-bénéfice qui ont été appliquées traditionnellement dans le cadre des politiques régionales. Les contributions mentionnées considèrent que l'analyse de ces politiques doit adopter un caractère systémique, en évitant de se focaliser sur les aspects superficiels de leur mise en œuvre, pour analyser le contexte institutionnel, les mécanismes profonds et la véritable nature des relations qui expliquent la dynamique de ces systèmes (Doloreux, Parto 2005 ; Sugden, Wei, Wilson, 2006). 
En s'arrêtant sur l'analyse de ces relations, leur apparition, leurs interactions, leurs impacts, etc., on génère une série de contributions autour du concept de gouvernance. En suivant les contributions pionnières de Storper et Harrison (1991) et Markusen (1996) pendant la dernière décennie, de nombreux travaux ont appliqué le concept de gouvernance, en tentant d'approfondir la dynamique des conglomérats productifs et des programmes qui prétendent les promouvoir (de Propris, 2001 ; de Propris, Wei, 2007 ; Aranguren et al., 2008 ; Parrilli, Sachetti, 2008 ; Provan, Kenis, 2008 ; Arikan, Schilling, 2011 ; Clifton et al., 2011 ; Bocquet, Mothe, 2010). D'après cette ligne de recherche, la gouvernance analyse la dichotomie entre le pouvoir et la confiance, entre la hiérarchie et la réciprocité, jusqu'à déterminer la nature des réseaux de production (Amin, 1999 ; Bachmann, 2003 ; Christopherson, Clark, 2007). Ainsi, derrière l'apparence d'uniformité pouvant exister dans un réseau, avec une structure et une organisation pour la production, il existe une grande variabilité, comme résultat des différentes formes de répartition du pouvoir entre les membres des réseaux, et des différentes règles régissant leurs relations avec les autres, c'est-à-dire, résultant de ses différentes possibilités d'exercer la gouvernance. C'est pourquoi on arrive à la conclusion qu'à la différence des approches orthodoxes, le pouvoir n'est pas le résultat de l'échec du marché, mais plutôt un élément constitutif des relations économiques; en conséquence, "il n'y a pas d'interaction sans pouvoir » (Sacchetti, Sugden, 2003). C'est pourquoi ce qui compte dans la détermination du réseau de production, et de son modèle explicatif, ce ne sont pas les attributs structurels (par exemple, le nombre d'acteurs, la taille, la concentration) mais la personne qui prend les décisions stratégiques et la manière dont celles-ci sont adoptées.

\section{LE PROGRAMME DE SOUTIEN AUX GEI}

Dans le cas précis de la politique industrielle espagnole, pendant une grande partie de son histoire, ses actions et ses intérêts étaient loin des considérations territoriales; ils étaient axés sur les aspects sectoriels. Exceptionnellement, et de manière ponctuelle, on peut citer les Plans de développement des années soixante ou le soutien aux Zones de réindustrialisation urgente (connues comme les ZUR) du milieu des années 1980, qui ont fait l'objet de quelques actions singulières d'une certaine importance au niveau territorial (Ybarra, 2009). À partir de là, le processus de décentralisation politique intense qu'a connu l'État espagnol a eu comme résultat le transfert aux Communautés autonomes des compétences en matière de promotion industrielle à caractère territorial. Ainsi, dans le contexte de la 
politique industrielle espagnole, un fait assez nouveau et d'une importance spéciale est l'adoption de mesures qui pariaient fortement sur une politique industrielle à base territoriale.

Plus récemment, cette impulsion s'est concrétisée en 2006 par la mise en place d'un programme extraordinaire pour créer des Groupements d'Entreprises Innovantes $(\mathrm{GEI})^{2}$ ayant pour objet d'encourager la création de groupes d'entreprises qui pourraient déclencher une dynamique sectorielle et territoriale pour la croissance et l'innovation, favorisant l'apparition de clusters et/ou de districts industriels (Trullén, 2009). Cette nouvelle initiative vise à identifier et à encourager un nombre suffisant d'entreprises, basées sur certains territoires et activités, afin d'obtenir une masse critique minimale, capable de créer des dynamiques d'innovation dans les activités et les secteurs retenus, qu'ils soient traditionnels ou avancés, dans l'industrie espagnole. L'importance de ce programme ne réside pas uniquement dans la création d'une capacité de changement et de transformation, mais aussi dans le fait que sa conception est basée expressément sur les concepts théoriques mentionnés plus haut des districts industriels et des clusters. L'accent est mis sur la valorisation des variables territoriales pouvant surgir, comme éléments décisifs pour le développement des groupes d'entreprises.

Les GEI se définissent comme "la combinaison, dans un espace géographique ou secteur productif concret, d'entreprises, centres de formation, unités de recherche publique ou privée, et autres agents publics ou privés, intégrés dans un processus de coopération leur permettant d'obtenir des avantages ou des bénéfices dérivés de l'exécution de projets conjoints à caractère innovateur et d'obtenir une masse critique telle qu'elle permette d'assurer leur compétitivité internationale et leur visibilité ». Par conséquent, la notion des GEI se rapproche de la notion de "pôle de compétitivité » (cluster innovateur) reprise dans l'encadrement communautaire des aides de l'État à la $R \& D$. L'aide instrumentale proposée se compose de subventions accordées aux groupes, associations et/ou entités à but non lucratif dont les caractéristiques sont proches de ce qui est défini comme le GEI, ou le cas échéant, qui agissent en tant que promoteurs de ces organismes. Instrumentalement, les aides accordées dans le cadre du programme sont limitées à quatre modalités de projets : élaboration de plans stratégiques, mise en œuvre des structures de coordination du GEI, réalisation d'activités spécifiques visant à renforcer le potentiel innovant des

2. Voir les arrêtés du ministère de l'Industrie, du Commerce et du Tourisme ITC/2691/2006, du 2 août, ITC/1843/2009, du 3 juillet, IET/1600/2012, du 16 juillet, régulant les bases, le régime d'aides et la gestion des mesures de soutien aux groupements d'entreprises innovantes. 
entreprises intégrées au sein du GEI et, finalement, réalisation de projets de coopération entre différents GEI. ${ }^{3}$

Comme résultat du programme, un total de 165 GEI a été incorporé au Registre spécial de GEI, créé à cet effet par le ministère espagnol de l'Industrie. Pendant cette période, le financement accordé par le Programme de soutien aux GEI non touristiques s'est élevé à 32,4 millions d'euros. À l'origine, le financement était destiné pour la plupart aux deux premières modalités de soutien, la préparation des plans stratégiques ( 9 millions d'euros) et la mise en place de structures de gestion (12,2 millions), alors que les modalités d'activités spécifiques et de projets de coopération ont reçu un financement moindre (11,2 millions d'euros dans l'ensemble). La logique de cette distribution était expliquée par le besoin initial de favoriser la création de GEI et doter le programme de la masse critique d'associations nécessaire pour son fonctionnement. Lors des appels successifs, notamment avec la suppression en 2010 du financement à la mise en place de plans stratégiques, la distribution des ressources du programme entre les différentes modalités montre une tendance à l'équilibre.

Les indicateurs financiers exposés doivent être pris en compte pour déterminer la portée réelle du programme de GEI. Ainsi, le coût annuel moyen du programme de GEI se situe à quelque 6,5 millions d'euros qui représentent environ $4 \%$ du coût total destiné annuellement par le ministère de l'Industrie au soutien aux PME et 10 \% du coût non financier (chiffres du Budget général de l'État pour 2011). Si l'on adopte le critère de l'accumulation financière, la capacité directe de mobilisation du programme devient très limitée en raison des pourcentages élevés de co-financement prévus, qui peuvent s'élever en valeurs absolues et en fonction de la modalité du projet entre $75 \%$ et $60 \%$. Quant aux pourcentages effectifs de co-financement, en se rapportant aux chiffres pour la période 2007-2011 (MITYC, 2013), le programme de GEI a obtenu une mobilisation directe de ressources financières privées d'une valeur totale de 60,3 millions d'euros avec un financement public de 32,5 millions d'euros. Par conséquent, en tenant compte uniquement de la mobilisation directe de ressources, l'accumulation financière du programme est limitée.

En ce qui concerne la finalité du soutien apporté, dans un premier temps nous pouvons classer les GEI qui ont vu le jour en répondant aux critères du ministère de l'Industrie lui-même, et qui ont déjà été employés dans d'autres travaux (Trullén, Callejón, 2008) en quatre groupes : a) « districts industriels

3. Les aspects relatifs à l'instrumentation et à l'application du programme de soutien aux GEI peuvent être consultés plus en détail dans les publications de Trullén et Callejón (2008), Ybarra et Doménech-Sánchez $(2011,2012)$. 
marshalliens » intégrés par des concentrations territoriales de petites entreprises spécialisées dans un secteur (activité) particulier ; b) les « chaînes de valeur » dans lesquelles un groupe de petites et moyennes entreprises fournisseuses distribue des inputs à des entreprises de dimension supérieure ; c) « activités intensives en connaissance » intégrées par des entreprises à intensité de recherche qui commercialisent les produits surgis de procédés innovateurs ; et d) « activités intensives en TIC - technologies de l'information et de la communication » qui s'appuient sur les technologies de l'information pour créer ou commercialiser leur gamme de produits ou de services. Une dernière catégorie a été ajoutée à ce classement, regroupant les GEI dans le secteur du tourisme. Le tableau 1 montre la distribution des 165 GEI inscrits sur le registre au début de l'année 2011.

\section{Tableau 1 - Distribution des GEI selon les caractéristiques du groupement d'entreprises}

\begin{tabular}{|l|c|c|c|c|}
\hline & \multicolumn{2}{|c|}{2009} & \multicolumn{2}{c|}{2011} \\
\hline & Nombre & $\%$ & Nombre & $\%$ \\
\hline a. Districts industriels & 18 & 17,8 & 30 & 18,2 \\
\hline b. Chaînes de valeur avec des entreprises tractrices & 24 & 23,8 & 38 & 23,0 \\
\hline c. Activités basées sur la connaissance & 20 & 19,8 & 23 & 13,9 \\
\hline d. Activités industrielles et de services intensifs en TIC & 11 & 10,9 & 25 & 15,2 \\
\hline e. Tourisme & 21 & 20,8 & 31 & 18,8 \\
\hline Autres & 7 & 6,9 & 18 & 10,9 \\
\hline Total GEI & 101 & 100 & 165 & 100 \\
\hline
\end{tabular}

Secteurs compris dans chaque groupe : a) Agro-alimentaire, santé, céramique, chaussure, textile et mode, bois, meuble et bouchons, robinetterie, emballage, jouets ; b) Automobile, ferroviaire, naval, machines, transport ; c) Aéronautique, biotechnologie, eau et énergie, environnement, optique ; d) Média, TIC, arts graphiques et design ; e) Tourisme.

Source : Ministère de l'Industrie $(2010 ; 2011)$.

Les résultats de ce classement montrent que seulement $18 \%$ des GEI enregistrés correspondent au concept traditionnel de districts industriels marshalliens dans lequel la proximité géographique et la connaissance productive partagées facilitent l'obtention d'économies externes et, en conséquence, l'amélioration de la productivité du groupement territorial de petites et moyennes entreprises. Les groupements encadrés dans la catégorie de chaînes de valeur et formés par des groupements qui correspondent au modèle de «cluster monopsonistique » ont un poids comparable dans le total (23\%), bien que dans ces industries en général, les entreprises soient situées dans la même région/État, le territoire perd le caractère d'épine dorsale du processus productif, car il s'agit d'activités dans lesquelles la chaîne de valeur peut se traduire par des réseaux de fourniture à caractère national ou international, et où la hiérarchie entre les entreprises prime sur toutes les 
autres caractéristiques. Jusqu'ici il semble qu'il existe encore un véritable équilibre entre les conceptions théoriques traditionnelles dans lesquelles la variable régionale et territoriale est présente, ce qui est compatible avec la proposition pratique suivant laquelle la composante sectorielle est celle qui dicte la détermination du GEI. Cependant, cet équilibre est relatif du fait qu'une grande partie des GEI restants sont principalement sectoriels par rapport au territorial. Ainsi, dans les GEI dont les activités sont basées sur la connaissance ( $14 \%$ du total) et sont intensifs en TIC (15\%), en raison des caractéristiques du processus productif et innovant propre à ces secteurs, on observe des groupements où priment les relations horizontales entre les entreprises dans des domaines tels que les ventes, le marketing ou la $R \& D$, par rapport à la verticalité des relations caractéristiques marshalliennes de districts industriels. Nous devons faire une mention spéciale pour le secteur de l'aviation à cause de la hiérarchie des relations verticales entre les différentes phases de production similaires à celles indiquées pour le groupe des sociétés créant des chaînes de valeur (entreprises locomotives). Enfin, les groupes du secteur touristique ont été incorporés au registre en 2009, en réponse à l'intérêt exprimé par le ministère de l'Industrie, du Commerce et du Tourisme de prioriser les projets innovants dans ce secteur en réponse aux nouvelles possibilités offertes par les initiatives communautaires.

Malgré la proposition initiale de départ, le lien des GEI avec leur territoire est très relatif, restant à l'arrière-plan. Ce sont les aspects relatifs à l'innovation en général qui déterminent la génération de ces groupements. On pourrait affirmer que l'innovation, indépendamment du lieu où elle se produit, est la variable qui génère la création des GEI. Ainsi, en classant les objectifs pour lesquels les GEI sont créés, nous avons le tableau suivant (tableau 2).

Tableau 2 - Activités à développer par les GEI (\% du total de GEI)

\begin{tabular}{|l|c|}
\hline Activité à développer & $\%$ \\
\hline $\begin{array}{l}\text { Communication externe sur le cluster / Amélioration de l'image et visibilité du } \\
\text { secteur }\end{array}$ & 97,6 \\
\hline Coordination mobilisation des agents du secteur au niveau territorial. & 78,0 \\
\hline Soutien à la participation des associés à des projets ou des programmes de R+D & 75,6 \\
\hline Formation des ressources humaines & 68,3 \\
\hline Organisation de rencontres sectorielles & 63,4 \\
\hline
\end{tabular}

Source : Compilé à partir des informations fournies par les $\mathrm{GEI}^{4}$

4. Les tableaux 2 et 3 ont été élaborés à partir de différentes sources complémentaires talles que les publications et les sites web élaborées par les GEI, ou les résultats des enquêtes citées dans des rapports d'évaluation récents publiés par le MICYT (2010, 2011). 
Bien qu'il soit quelque peu prématuré, car le programme est toujours en vigueur, il convient de noter que les activités dans lesquelles les GEI créés sont principalement ancrés sont celles liées au développement de projets d'innovation. Les actions qui ressortent sont celles relatives à la communication d'une image vers l'extérieur, la coordination et la mobilisation des personnes et des institutions du territoire, la canalisation des efforts collectifs visant à la participation à des projets que chaque partenaire ne pourrait pas aborder séparément, la formation de personnel qualifié et la réalisation d'actions de sensibilisation dirigées vers l'ensemble du secteur en question. On remarque ainsi, dans les GEI créés, le caractère novateur, en général, face à la variabilité territoriale et le phénomène d'entraînement qui pourrait en découler. L'association au sein du GEI est soumise à la collecte de fonds rendant possible le projet. L'image et la publicité faite autour du projet sont valorisées en premier.

\section{LA GOUVERNANCE DANS LE CADRE DES GEI}

L'impact des GEI sur leur environnement à des degrés divers et l'obtention de leurs objectifs ultimes dépendra, dans une large mesure, de leur capacité à faire participer et rendre responsables les différents agents et sujets du territoire appartenant à des secteurs disparates, face aux actions dictées par les autorités centrales, et souvent sans rapport avec la réalité territoriale. C'est pourquoi l'étude du processus de façonnement des GEI, ainsi que l'identification des mécanismes qui les régissent, permettra d'approfondir le résultat final que l'on peut attendre de leur mise en œuvre. Par conséquent, il est nécessaire d'analyser plus en profondeur les aspects de la gouvernance des GEI.

En reportant la discussion sur le modèle de gouvernance et ses implications pour le cas des GEI espagnols, il convient de noter que les actions contenues dans le Programme de soutien donnent la priorité aux mesures visant à renforcer et promouvoir les structures de gouvernance qui permettent aux groupes de canaliser les ressources productives vers l'amélioration de la situation concurrentielle locale. Cela découle du fait que la plupart des ressources fournies au cours des trois appels effectués à ce jour ont eu pour but de soutenir la planification stratégique et la mise en œuvre des structures de gestion, avec un soutien plus limité pour les autres actions prévues (activités et projets spécifiques en matière de coopération).

Les règles qui régissent le programme permettent d'obtenir le statut de GEI par des organismes ayant des caractéristiques très différentes au départ. Il est seulement nécessaire que les entités aient une personnalité juridique 
distincte, qu'elle corresponde à une entité à but non lucratif, que sa nature et les objectifs soient cohérents avec ceux mis en place dans la définition du GEI et que les plans stratégiques présentés aient obtenu le qualificatif d'excellent. Par conséquent, il se pourrait que des GEI promus par des entités aussi diverses que les centres de recherche coopérative privés, les associations d'affaires, les fondations publiques ou privées, les universités et les municipalités demandent l'enregistrement en tant que GEI. Cette diversité complique l'analyse des informations disponibles sur les groupes enregistrés, mais certains indicateurs peuvent être utilisés pour établir à peu près la structure organisationnelle existante et la relation entre les acteurs qui les composent. Ces indicateurs sont regroupés en deux catégories : d'abord, les caractéristiques structurelles et sur l'origine du groupe, et d'autre part, les éléments se rapportant au mode de gouvernance. Parmi les premiers, tout en tenant compte de la taille moyenne des entreprises au sein de chaque groupement, l'intensité technologique et la portée géographique, il faut également tenir compte de la préexistence d'une structure d'interaction entre les entreprises et les institutions comprises dans le groupe et de l'origine du promoteur qui a assumé un leadership initial. Parmi les indicateurs qui définissent le modèle de gouvernance, on peut envisager l'asymétrie dans la distribution du pouvoir de décision, les caractéristiques des organes de gestion du groupement, le milieu dans lequel les décisions stratégiques et opérationnelles sont adoptées et les mécanismes d'action et d'apprentissage collectif.

En ce qui concerne le premier indicateur, la taille des entreprises qui font partie du GEI, nous voyons qu'elle dépasse la taille moyenne de l'industrie espagnole (tableau 3). En termes de nombre de salariés, la dimension moyenne des entreprises des GEI non touristiques est de 198,7 travailleurs, alors que pour l'ensemble du pays la dimension moyenne des entreprises est de 16,1 salariés pour l'ensemble des entreprises industrielles et de 22,8 pour les entreprises industrielles et de services à haute et moyenne-haute technologie ${ }^{5}$. Cette grande différence entre la dimension des membres du groupement et la caractéristique des entreprises de l'ensemble de l'économie espagnole s'accentue dans les groupements d'entreprises encadrés comme des chaînes de valeur avec des entreprises tractrices, intensives en connaissance ou intensives en TIC (groupes b, c, d du tableau 1).

5. Enquête Industrielle d'Entreprises 2009 et Indicateurs de Haute Technologie 2009 (Institut national de statistique). 
Tableau 3 - Dimension moyenne des entreprises participant au programme de GEI

\begin{tabular}{|l|c|c|c|}
\hline & Employés & Entreprises & $\begin{array}{c}\text { Taille } \\
\text { moyenne }\end{array}$ \\
\hline 1. Districts industriels & 36908 & 361 & 102,2 \\
\hline 2. Chaînes de valeur avec entreprises locomotives & 416972 & 1794 & 232,4 \\
\hline 3. Activités basées sur la connaissance & 243689 & 792 & 307,7 \\
\hline 4. Activités industrielles et de services intensifs en TIC & 88487 & 226 & 391,5 \\
\hline
\end{tabular}

Source : Compilé à partir des informations fournies par le GEI.

Un deuxième indicateur est la mesure et les caractéristiques des liens antérieurs existant entre les entités qui constituent les GEI. Dans certains cas, le GEI part d'un groupement d'entreprises et/ou institutions préexistantes ayant une trajectoire consolidée, qui se présentent au programme dans le but d'élargir leurs possibilités ou leur moyen d'action. Dans d'autres cas, le groupement est configuré et institutionnalisé afin de répondre à l'appel et d'obtenir l'enregistrement, en définissant ses objectifs et son organisation à partir du plan stratégique. Enfin, il existe des situations dans lesquelles à partir d'un ou plusieurs groupements et institutions déjà existantes le GEI est créé avec un véritable degré d'autonomie à l'égard des fondateurs et avec des objectifs propres. L'étude du moment de la création des organisations qui demandent l'enregistrement comme GEI met en évidence que le soutien aux groupements s'est adressé aussi bien à la stimulation de la création de groupements d'entreprises $(45,9 \%)$ qu'à la consolidation de groupements déjà existants sous une autre forme juridique $(54,1 \%){ }^{6}$

De même, les caractéristiques de l'organisation promotrice du GEI peuvent aussi apporter des informations importantes sur le lien qui s'établit entre les entreprises et les institutions qui constituent le réseau de relations du groupement, dans la mesure où cela va conditionner quelques aspects clef de son fonctionnement tels que les objectifs, le financement ou les projets dans lesquels vont s'impliquer les organisations associées. Selon les informations fournies par le MICYT (2010), la majeure partie des initiatives sont promues par des groupements d'entreprises à eux seuls (32\%), ou bien en collaboration avec d'autres agents publics (40 \%). Un nombre plus réduit de

6. Cette donnée présente quelques difficultés dans son obtention car tous les groupements ne sont pas repris. En premier lieu, quelques groupements ne fournissent pas ces informations sur leurs sites web ou dans la documentation publiée. Dans d'autres cas, lorsqu'il y a un partenaire qui joue un rôle clé dans la promotion et le fonctionnement du GEI, il y a une certaine discrétion dans la décision d'assigner la date de création de l'établissement promoteur ou de la création du GEI. Dans ce dernier cas, en général, on a opté pour refléter celle de l'établissement promoteur, puisque celle-ci va conditionner le modèle d'organisation et d'interaction du groupement. 
GEI a été promu par l'administration (20\%) ou par le milieu de la recherche (8\%). Une analyse plus détaillée que nous avons réalisée sur les GEI dont on dispose d'informations confirme le poids qu'ont les associations d'entreprises dans leur promotion, bien que dans de nombreux cas, ces initiatives comptent également avec la participation active des autorités régionales, locales et autres agents du système d'innovation régional. Parmi les initiatives développées en collaboration entre les secteurs public et privé, on peut souligner le rôle joué par des centres de technologie dans leur promotion, ce qui est particulièrement vrai dans les Communautés autonomes où il y avait une structure préexistante régionale de ce type d'installation. Des paradigmes de ce modèle sont les cas du Pays Basque ou de la Communauté de Valence, où les centres technologiques dirigent nombre de ces initiatives et participent de façon significative. Dans les initiatives mises en place, on peut attribuer un rôle important à la collaboration des fondations et des associations à but non lucratif, qui ne sont pas comprises dans la catégorie de centre technologique ; et parmi elles on peut trouver un bloc important dans lequel se trouvent des fondations semi-publiques, des établissements à but non lucratif ayant des objectifs génériques de promouvoir l'innovation et la connaissance, ou des fondations à participation publique et privée créées dans le but exprimé de répondre à l'appel de soutien aux GEI. Enfin, les cas dans lesquels l'administration a acquis le rôle principal dans la mise en œuvre des initiatives sont très liés à ces Communautés Autonomes dans lesquelles les gouvernements régionaux ont adopté une stratégie uniforme et prédestinée de promotion des clusters, de sorte que, initialement, les autorités régionales dirigent tous les GEI constitués ; tel est le cas de la Communauté de Madrid dans laquelle tous les GEI sont groupés sous l'initiative « parapluie » Madrid-Network, en partageant ainsi des coûts et des ressources communes. Enfin, les données attribuent un rôle réduit aux universités dans la promotion des GEI.

Mais quelles que soient les caractéristiques structurelles des clusters, il est essentiel de connaître le modèle de développement économique et institutionnel émergent qui vient d'être établi entre les participants. Des aspects tels que la répartition du pouvoir, les caractéristiques des organes de gestion et le niveau auquel les décisions sont stratégiques et opérationnelles, permettent de déterminer, entre autres, les structures de gouvernance et de son évolution possible. Compte tenu de ces variables comme étant des axes, nous pouvons identifier une variété de modèles de gouvernance, mais, comme indiqué par Grabher et Powell (2004, p. XVII-XVIII) dans l'analyse des réseaux de gouvernance " ces formes ne sont pas des catégories distinctes identifiées ou essentialistes mais, plutôt, elles se chevauchent et s'emboîtent à des degrés différents. Les formes particulières de réseau évoluent comme 
des combinaisons complexes de chevauchement des mécanismes de gouvernance, juxtaposées et imbriquées ». Voici les résultats de cette analyse où nous avons identifié quatre types des GEI.

Dans les groupements identifiés sous le modèle de « cluster monopsonistique », on reconnaît clairement l'impulsion des grandes entreprises dans leur formation. Dans des secteurs comme l'automobile ou l'aéronautique, les réseaux de fournisseurs découlant de la décentralisation du processus productif des grandes entreprises ont consolidé des groupements localisés d'entreprises de taille moyenne et petite. Selon la typologie bien connue de Markusen (1996), ces groupements répondraient initialement au modèle hub-and-spoke dans lesquels les entreprises leaders, de plus grande taille, ont un rôle principal déterminant dans la génération des conditions productives et technologiques du cluster (liens commerciaux avec l'extérieur, formation des ressources humaines, création d'entreprises spin-off,...). Souvent, le développement du cluster à partir de ces entreprises leaders consolide des relations entre les agents du groupement pour être institutionnalisées par le biais de partenariats avec des entreprises établies et la projection au-delà du niveau local. Ces associations, qui comptent déjà une certaine représentativité dans le secteur, envisagent leur constitution en GEI comme une manière de consolider leur position de référence et comme un moyen d'accéder à des voies de financement additionnelles. Les cas du cluster de l'Aéronautique et de l'Espace du Pays Basque (HEGAN), de l'Association Valencienne de l'Industrie de l'Automobile (AVIA) ou du cluster d'Entreprises d'Automobile de la Galice (CEAGA), sont également, représentatifs de ce modèle. La contribution du programme de GEI à la gouvernance de ces groupes devra être évaluée en termes de leur capacité à créer des liens entre les membres du cluster moins hiérarchiques. En somme, la création du GEI doit jouer un rôle de coordination dans la transition d'un modèle de relations hiérarchiques à une autre "hétérarchie" dans lequel les petites entreprises participent à l'adoption des décisions stratégiques déterminantes dans l'évolution du cluster. ${ }^{7}$

Parmi les groupes qui ont leur origine dans des associations professionnelles on trouve le groupe GEI représentant le modèle de district industriel marshallien. Dans ce cas, les relations entre les entreprises locales du système de production ont consolidé les partenariats établis de longue date dans le territoire qui exercent des fonctions qui vont au-delà de la représentation des intérêts collectifs de leurs membres. Ce modèle est également présent parmi les autres secteurs dans le secteur de l'industrie alimentaire, du bois

7. On peut aussi intégrer dans ce modèle quelques-uns des GEI créés dans le secteur maritime qui s'appuient sur les réseaux d'entreprises et d'associations surgies par le passé pour la protection des chantiers navals publics ou la tradition de la pêche de la zone. 
et des machines. Ces associations peuvent compter, entre autres, avec des services comme des centres de recherche coopérative, des systèmes de certification des entreprises associées ou des centres de formation. ${ }^{8}$ Dans ces cas, le GEI se superpose à une structure dans laquelle l'opération est identifiée, à la fois en service comme dans les entreprises partenaires. Par conséquent, la création du GEI joue un rôle stimulant des activités déjà en cours d'élaboration, même si, dans cet environnement d'association de petites et moyennes entreprises et donc du GEI, elle a un rôle clé en tant que lien entre les agents du système productif local sur les marchés étrangers et des flux de technologie. Les GEI permettraient aux entreprises à système de production local d'identifier, d'acquérir et d'utiliser les connaissances générées à l'étranger et seraient donc une institution clé pour faciliter la maturation du cluster.

Dans certains de ces groupements qui se rapprochent du modèle de district industriel marshallien, les centres de recherche ont eu un rôle stimulant dans la formation et le développement du cluster comme dans la création ultérieure du GEI. Les centres de recherche coopérative font partie du réseau et contribuent autant à lier entre elles les entreprises de l'agglomération qu'à canaliser vers le système local les flux de connaissance extérieurs à celui-ci. Par conséquent, il ne faut pas confondre cette situation avec les groupements promus et coordonnés depuis des institutions publiques (qu'elles soient ou non de recherche) dont il sera question plus tard.

Certains de ces centres proviennent d'associations d'entreprises déjà établies, d'autres sont créés par les universités techniques et, dans certains cas, l'initiative vient des politiques de développement industriel des administrations régionales. La nature différentielle de ces pôles est due à l'infrastructure de recherche collective qui tente de coordonner et de mobiliser les ressources locales à travers divers mécanismes tels que, notamment, le transfert de technologie, la formation des ressources humaines, les mécanismes de surveillance technologiques et le soutien à la création de nouvelles entreprises. Les groupes créés selon le principe de l'Institut technologique de Valence sont représentatifs de ce modèle dans lequel le centre de technologie a un rôle actif dans la promotion et le développement de groupements du plan stratégique du GEI avec les entreprises et les associations représentant le secteur (cluster d'innovation Chaussures, le secteur GEI carreaux de céramique, ou AIDIMA).

Face aux modèles antérieurs, pour ceux dont l'impulsion correspond à l'initiative privée, on a identifié d'autres groupements dans lesquels la

8. Dans ce groupe on peut inclure quelques associations d'entreprises constituées en GEI dont le milieu est tout le territoire national. 
présence des institutions ou des administrations a été déterminante sur la formation et le développement ultérieur du cluster. Parfois, la dépendance de l'initiative publique relève de la spécificité sectorielle et technologique de l'activité que l'on souhaite promouvoir. Dans d'autres cas, le soutien à la formation du cluster répond aux priorités des politiques de développement territorial. Parmi les GEI plus intenses en technologie et connaissance on peut mentionner les secteurs de la biotechnologie (par exemple, Bioregió de la Catalogne, Biotechnologie de la Communauté de Valence - BIOVAL, etc.), les médias (Barcelone ou Madrid plate-forme de médias audiovisuels) et les technologies de l'information et des communications. Ce sont tous des cas visant des groupes nouvellement créés dont la taille et la localisation sont très diversifiées institutionnellement (Fondation de l'Université, l'Institut technologique promu par l'administration régionale et/ou locale, fondation, association ou organisme privé avec une forte implication des institutions publiques). Le principal défi de ces GEI, est la nécessité d'évoluer pour passer d'un modèle de gouvernance publique, où les décisions stratégiques sont prises sur la base de critères d'opportunité scientifique et technologique, à un autre dans lequel les entreprises du groupement participent au processus de détermination des objectifs collectifs. ${ }^{9}$

Parmi ces groupements dans lesquels les administrations ont un rôle protagoniste dans leur formation, on en voit aussi apparaître quelques-uns liés à des secteurs de moindre intensité technologique tels que l'agro-alimentaire ou les machines (par exemple, le GEI Food+i ou Manufacturias GEI). Il est difficile d'établir une hypothèse sur l'évolution future de ces groupements. Par ailleurs, il faut attendre que le soutien public ait pour résultat un accroissement de la base technologique et des capacités du réseau d'entreprises et d'institutions qui constituent le groupement. En outre, il faut prévoir que le soutien aux activités et aux infrastructures très intensives en $R \& D$ pourrait détourner les ressources et les capacités des autres activités avec une plus grande potentialité commerciale (Lucas et al., 2009). Pour que les efforts initiaux se traduisent par une impulsion des capacités innovantes et commerciales il faut que l'initiative publique se tourne vers la création de nouvelles entreprises, l'ouverture de nouveaux marchés ou la commercialisation de nouveaux produits. Depuis la perspective de la gouvernance du réseau, il doit se produire une transition pour passer d'un modèle dans lequel les

9. Dans ce groupe on peut inclure aussi d'autres GEI comptant avec un fort soutien de l'administration dans leur processus de constitution et qui ont leur origine dans des services de réseau ou des services publics réglés (utilities) tels que l'eau, l'énergie ou le transport. Ces GEI se caractériseraient aussi par la forte participation de grandes entreprises ainsi que par le poids qu'ils ont sur les décisions stratégiques en ce qui concerne la projection publique d'une image collective du secteur. 
décisions tant stratégiques que concernant les opérations en matière d'innovation sont adoptées par l'administration (ou par les organes constitués pour stimuler le cluster), à un autre caractérisé par la répartition symétrique de la capacité de décision capable de générer un apprentissage dynamique. Ce processus de réaménagement de la structure de gouvernance ne pourra avoir lieu que si le cluster a la capacité de grandir à partir de la génération de nouvelles entreprises à travers des spin-off produits dans les institutions ou en attirant les entreprises du secteur ou des secteurs proches.

\section{ESTIMATIONS FINALES DU PROGRAMME DE GEI : IMPACT ET LIMITES}

Il convient de noter que la politique industrielle proposée dans le cadre des programmes d'autonomisation des GEI tente d'intégrer les variables spatiales et territoriales comme l'une des priorités de ses propositions. Implicitement, le potentiel de développement endogène des différents territoires et les capacités des économies externes impliquant les entreprises dans des territoires spécifiques, seront évalués implicitement dans le but de générer des processus de développement innovants. Cependant, malgré ces premières propositions, au fur et à mesure que les GEI sont créés, le lien qu'ils entretiennent avec leur territoire est relatif, ce principe étant relégué à l'arrière-plan. La réalité est que ce seront les aspects strictement liés à l'innovation qui seront déterminants pour la conception de ces grappes. On peut affirmer alors que c'est l'innovation, partout où elle se présente, la variable qui favorise la formation des GEI. La mise en œuvre du programme de GEI serait en fait l'aval théorique et pratique, le justificatif analytique et politique, pour appliquer effectivement une politique industrielle générique, dont l'objectif serait axé sur la promotion de nouvelles activités qui pourraient être considérées comme stratégiques dans l'économie mondiale (biologie, énergies, environnement, aéronautique, etc.).

Les clusters se caractérisent par leur orientation vers l'innovation, le caractère territorial devenant plus flou et étant relégué à un arrière-plan. Une deuxième constatation est que le programme GEI consolide les tendances existantes, renforcées dans la plupart des cas, les possibilités certaines et vérifiées, et dans d'autres cas, au moins, la découverte de nouvelles opportunités innovantes. C'est pourquoi, dans le cas espagnol de création des clusters, nous voyons que les procédures couvertes par le Programme de soutien font prévaloir des mesures pour renforcer et promouvoir les structures de gouvernance existantes et des groupements qui permettent de canaliser les ressources productives vers l'amélioration des conditions de concurrence. 
Par conséquent, la plupart des ressources fournies par le programme de subvention ont été conçues pour soutenir la planification stratégique et la mise en ouvre de structures de gestion préétablies. De même, un autre élément qui viendrait confirmer ce point de vue de la continuité du programme est le fait que plus de la moitié des GEI créés sous son égide sont basés sur des associations ou des groupements qui existaient déjà. C'est la promotion des associations d'entreprises, la volonté expresse des autorités régionales et locales, ainsi que certains agents des systèmes d'innovations régionaux qui seront au centre de la création des GEI. On peut noter l'absence relative des universités dans ce processus, ce qui est peut-être dû au fait que leur objectif est davantage axé sur les aspects de la recherche fondamentale plutôt que sur le développement des affaires.

Un troisième aspect à souligner est que les programmes de soutien à la production de GEI vident des groupes d'entreprises qui n'auraient peut-être pas besoin de concevoir un programme spécifique pour qu'une dynamique innovante se déroule. La réalité est que la taille des entreprises qui font partie des clusters, dépasse de loin la taille de la moyenne industrielle espagnole. Le résultat final du programme montre que la prétention d'être un outil utile pour les petites entreprises, tel qu'il a été conçu à l'origine, devient un outil quasi exclusif des grandes entreprises. Et par-dessus tout, dans la mesure où ce sont des grappes ayant une intensité en capital de plus en plus forte, la taille des entreprises de la grappe a tendance à être plus grande.

En ce qui concerne les structures de gouvernance (nouvelles relations économiques et institutionnelles établies entre les participants, distribution de puissance, caractéristiques et organismes de gestion des niveaux où les décisions sont stratégiques et opérationnelles), l'analyse a démontré un certain potentiel qui diffère en fonction des caractéristiques du modèle de gouvernance. Dans ces réseaux d'entreprises qui étaient dans une situation embryonnaire, le programme d'appui aux GEI peut être la promotion d'une identification des groupements dynamiques ou peut contribuer à leur consolidation. Cet effet est particulièrement pertinent à la lumière de l'orientation de la politique européenne sur l'innovation et sur les entreprises qui tendent à canaliser leurs ressources par le biais de groupements régionaux d'entreprises. En outre, les résultats des appels concernant les types de sociétés qui composent le GEI, montrent que la création de groupements peut aider à résoudre certains des déficits qui limitent les possibilités des entreprises dans des domaines tels que les réseaux, la coopération, la consolidation des flux de connaissances et de développement technologique et productif de R\&D. Les limitations de taille de l'entreprise et l'absence d'une masse critique de ressources et d'expertise sont parmi les principaux obstacles à la consolidation du GEI. 
Même dans les cas où il existait déjà un groupe de sociétés liées par la production d'une position verticale afin de consolider les structures organisationnelles formalisées par l'adoption d'une charte par le GEI, l'approbation des plans stratégiques a été en mesure de les aider à résoudre les " échecs de la gouvernance », en particulier dans les groupes dans lesquels le processus d'auto-organisation du groupement a échoué à identifier des objectifs collectifs, les conditions environnementales ou des procédures pour la coordination des organisations et des institutions. Le caractère historiquement dépendant des groupes de production territoriaux peut créer des modèles d'interaction entre les acteurs du cluster priorisant la hiérarchie par rapport à l'interdépendance, l'orientation à court terme et la concurrence par rapport à la coopération, ou le verrouillage par des actes routiniers qui limitent l'accès aux flux de technologie et de connaissances de l'extérieur face à l'innovation. Ainsi, le programme de soutien aux GEI pourrait non seulement contribuer à la création et la consolidation des groupes, mais générerait des changements dans les attitudes et les comportements des entreprises et des organisations. Dans les termes exprimés par l'OCDE (2006), il faudrait évaluer, par conséquent, la contribution du soutien du secteur public par sa capacité à interagir avec les stratégies et les comportements des participants du groupement (accumulation comportementale).

Dans les deux situations décrites, le groupement embryonnaire et de monopsone, le programme GEI peut aider à définir un modèle de gouvernance quand l'existence de procédures de coordination et d'interaction n'a pas été précédemment identifiée. Il est nécessaire de formaliser une structure de l'organisation du gouvernement et des fonctions de coordination du groupement, et de formuler un plan stratégique impliquant la hiérarchisation des objectifs et des actions à entreprendre par les entreprises et les institutions qui composent le cluster. Bien qu'il ne se soit pas nécessairement traduit par une amélioration de l'objectif ultime d'une compétitivité accrue, ce résultat est intéressant en soi étant donné l'orientation croissante des politiques européennes en faveur des pôles d'affaires. Ainsi, le pôle constitué en vertu du programme donnerait accès à d'autres programmes et mesures visant à soutenir les réseaux d'entreprises. Cette fonctionnalité articulant des initiatives et des projets pourrait être encore renforcée par la mise en œuvre du programme permettant l'incorporation de groupes ayant des caractéristiques très différentes en termes de territoire, le réseau de relations entre les participants, la taille de l'entreprise et l'intensité technologique.

Face à ces potentialités des programmes GEI on remarque également certains aspects instrumentaux qui auraient pu limiter l'efficacité du régime d'aides. Le poids faible accordé aux critères de territorialité de l'activité, 
donne lieu à une présence très importante de groupement fonctionnellement définis dans lesquels les grandes entreprises sont en mesure de conditionner le processus de prise de décisions. Dans d'autres cas, dans les territoires où, auparavant, il n'y avait pas de présence de réseaux d'entreprises, la création des GEI s'est trop appuyée sur des institutions très étroitement liées au secteur public de la recherche. Les travaux récents de Peck et Lloyd (2008) abordent l'impact négatif que l'omission des objectifs régionaux peut avoir sur l'efficacité des politiques de cluster, ce qui conduit à un manque d'intégration entre l'initiative du groupement et la stratégie économique globale de la région. Dans ces cas, les priorités du groupement seraient fixées suivant des objectifs nationaux, laissant ainsi les régions comme de simples « contenants » pour favoriser le changement. Cette approche contraste avec le potentiel de la grappe comme un mécanisme pour l'intégration des politiques et le ciblage de celles-ci dans une stratégie commune visant à améliorer la compétitivité (Porter, 2007).

L'absence d'un cadre pour l'exercice de la gouvernance multiniveaux dans le sens de la détermination collective pour soutenir les objectifs énoncés par Borras et Tsagdis (2008) s'est récemment traduite par la répartition sectorielle de l'aide et sa relation avec les réseaux économiques et la production réelle. L'analyse de la relation entre le groupement et la promotion territoriale, mène à la conclusion qu'il y a souvent un décalage entre les secteurs qui favorisent la spécialisation et les activités qui ont une plus grande capacité à générer de l'emploi et l'augmentation de la production. Mais en dehors des considérations territoriales, la question est de savoir si le soutien pour la planification stratégique dans ces secteurs a un effet potentiel sur le passage de modèles de gouvernance hiérarchiques, dominé par les grandes entreprises de la grappe, à d'autres hiérarchies, dans lesquelles les décisions stratégiques sont adoptées d'une manière participative. Dans la mesure où les actions prises dans le cadre du programme du GEI contribuent à ce processus de changement on peut prévoir dans ces clusters fonctionnellement définis, des processus d'identification des besoins et une amélioration continue des capacités collectives.

\section{REFERENCES}

AMIN, A. (1999), An institutionalist perspective on regional economic development, International Journal of Urban and Regional Research, 23 (2), 365-378.

ARANGUREN, M. J., ITURROZ, C., WILSON, J. R. (2008), Networks, governance and economic development: an introduction, in M. J. Aranguren, C. Iturroz, J. R. Wilson (eds), Networks Governance and Economic Development: Bridging Disciplinary Frontiers, Cheltenham, Edward Elgar, 1-18. 
ARIKAN, A. T., SCHILLING, M. A. (2011), Structure and governance in industrial districts: implications for competitive advantage, Journal of Management Studies 48(4), 772-803.

ASHEIM, B. T. (2003), Industrial districts: the contributions of Marshall and beyond, in Clark, G. L., Gertler, M. S., Feldman, M. P. (eds), The Oxford Handbook of Economic Geography, Oxford University Press, 413-431.

BACHMANN, R (2003), The Role of Trust and Power in the Institutional Regulation of Territorial Business Systems: Cooperation, Networks and Institutions in Regional Innovation Systems, Cheltenham, Edward Elgar.

BECATTINI, G. (1979), Dal 'settore' industriale al 'distretto' industriale. Alcune considerazioni sul l'unità d'indagine del l'economia industriale, Rivista di economia e política industriale, 1, 7-21.

BIANCHI, P., LABORY, S. (2006), From 'old' industrial policy to 'new' industrial development policies, in Bianchi, P., Labory, S. (eds) International Handbook on Industrial Policy, Cheltenham, Edward Elgar, 3-27.

BOCQUET, R., MOTHE, C. (2010), Knowledge governance within clusters: the case of small firms, Knowledge Management Research E Practice, 8(3), 229-239.

BORRAS, S., TSAGDIS, D. (2008), Cluster Policies in Europe: Firms, Institutions and Governance, Cornwall, UK.

BRUSCO, S. (1982), The Emilian model - productive decentralization and social integration, Cambridge Journal of Economics, 6(2), 167-84.

CHRISTOPHERSON, S., CLARK, J. (2007), Power in firm networks: What it means for regional innovation systems, Regional Studies, 41(9), 1223-1236.

CLIFTON, N., DAVID, R. EHRET, O., PICKERNELL, D. (2011), An analysis of actual and potential clustering structures, stakeholder governance activities and cross-locality linkages in the Welsh aerospace industry, European Planning Studies, 19(2), 279-309.

COOKE, P. (1992), Regional innovation systems: competitive regulation in the new Europe, GeoForum, 23, 365-382.

COWLING, K., OUGHTON, C., SUGDEN R. (1999), A reorientation of industrial policy? horizontal policies and targeting industrial policy, in K Cowling (ed.) Industrial Policy in Europe: Theoretical Perspectives and Practical Proposals, London, Routledge, 17-34.

DIEZ, M. A. (2001), The evaluation of regional innovation and cluster policies: Towards a participatory approach, European Planning Studies, 9(7), 907-923.

DE PROPRIS, L. (2001), Systemic flexibility, production fragmentation and cluster governance, European Planning Studies, 9(6), 739-753.

DE PROPRIS, L., WEI, P. (2007), Governance and competitiveness in the Birmingham jewellery district, Urban Studies, 44(12), 2465-2486.

DEI OTTATI, G. (1994), Trust, interlinking transactions and credit in the industrial district, Cambridge Journal of Economics, 18(6), 529-546.

DOLOREUX, D., PARTO, S. (2005), Regional innovation systems: Current discourse and unresolved issues, Technology in Society, 27(2), 133-153.

ETZKOWITZ, H., LEYDESDORFF, L. (2000), The Dynamics of Innovation: From National Systems and 'Mode 2' to a Triple Helix of University-Industry-Government Relations, Research Policy, 29, 109-123. 
FORAY, D. (2004), The Economics of Knowledge, Cambridge M.A., The MIT Press.

FROMHOLD-EISEBITH, M., EISEBITH, G. (2008), Looking behind facades: Evaluating effects of (automotive) cluster promotion, Regional Studies, 42(10), 1343-1356.

GIBBONS, M., LIMOGES, C., NOWOTNY, H., SCHWARTZMAN, S., SCOTT, P., TROW, M. (1994), The New Production of Knowledge: The Dynamics of Science and Research in Contemporary Societes, London, Sage Publications.

GRABHER, G., POWELL, W. W. (2004), Introduction: Networks Vol. I, in G. Grabher, W. W. Powell (eds), Networks: Vol I, Cheltenham, Edward Elgar Publishing, xi-xxxi.

HOSPERS, G., SAUTET, F., DESROCHERS, P. (2008), Silicon somewhere: is there a need for cluster policy?, in Ch. Karlsson (ed.), Handbook of Research on Innovation and Clusters: Cases and Policies, Cheltenham, Edward Elgar Publishing, 430-446.

LUCAS, M., SANDS, A., WOLFE, D. A. (2009), Regional Clusters in Global Industry: ITC Clusters in Canada, European Planning Studies, 17(2), 179-187.

MAILLAT, A., QUEVIT, M., SENN, L. (1993), Reseaux d'innovation et milieux innovateurs: un pari le développement régional, Nuechatel, GREMI-EDES.

MARKUSEN, A. (1996), Sticky places in slippery space: A typology of industrial districts, Economic Geography, 72(3), 293-313.

MARSHALL, A. (1920), Principles of Economics (8th edition), London, Macmillan.

MARTIN, R., SUNLEY, P. (2003), Deconstructing clusters: chaotic concept or policy panacea, Journal of Economic Geography, 3(1), 5-35.

MASKELL, P., MALMBERG, A. (1999), Localised learning and industrial competitiveness, Cambridge Journal of Economics, 23(2), 167-185.

MITYC (2013), El programa de AEIs 2007-2013. Balance actualizado de cinco años de aplicación de la política de clusters del MITYC, Dirección General de Política de la Pequeña y Mediana Empresa, MITYC, Madrid, http://www.ipyme.org/es-ES/publicaciones/Paginas/ Publicaciones.aspx

OCDE (2006), Government RED Funding and Company Behaviour: Measuring Behavioural Additionality, OCDE, Paris.

PARRILLI, M. D., SACCHETTI, S. (2008), Linking learning with governance in networks and clusters: key issues for analysis and policy, Entrepreneurship $\mathcal{E}$ Regional Development, 20(4), 387-408.

PECK, F., LLOYD, C. E. (2008), Cluster Policies and Cluster Strategies, in Karlsson, Ch. (ed.) Handbook of Research on Innovation and Cluster, Cheltenham, Edgar Elgar.

PIORE, M. J., SABEL, C. F. (1984), The Second Industrial Divide, New York, Basic Books.

PONDS, R., VAN OORT, F., FRENKEN, K. (2007), The geographical and institutional proximity of research collaboration, Papers in Regional Science, 86(3), 423-443.

PORTER, M. E. (1998), On Competition, Boston, MA, Harvard Business School Press.

PORTER, M. E. (2007), Cluster and Economic Policy: Aligning Public Policy with the New Economics of Competition, Institute for Strategy and Competitiveness, White Paper Nov. 2007.

PROVAN, K. G., KENIS, P. (2008), Modes of network governance: Structure, management, and effectiveness, Journal of Public Administration Research and Theory, 18(2), 229-252. SACCHETTI, S., SUGDEN, R. (2003), The governance of networks and economic power: The nature and impact of subcontracting relationships, Journal of Economic Surveys, 17(5), 669-692. 
STORPER, M., HARRISON, B. (1991), Flexibility, hierarchy and regional development: The changing structure of industrial production systems and their forms of governance in the 1990s, Research Policy, 20(5), 407-422.

SUGDEN, R., WEI, P., WILSON, J. R. (2006), Clusters, governance and the development of local economies: A framework for case studies, in R. Sugden, C. Pitelis, J. R. Wilson (eds), Clusters and Globalisation: The Development of Urban and Regional Economies, Cheltenham, Edward Elgar, 61-95.

TRULLÉN, J. (2009), National industrial policies and the development of industrial districts: reflections on the Spanish case, in G. Becattini, M. Belandi, de Propris L., Handbook of Industrial Districts, Cheltenham, Edgard Elgar, 726-738.

TRULlÉN, J., CALLEJÓN, M. (2008), Las agrupaciones de empresas innovadoras, Mediterraneo Económico, 13, 459-478.

VISSER, E. J., ATZEMA, O. (2008), With or without clusters: Facilitating innovation through a differentiated and combined network approach, European Planning Studies, 16(9), 1169-1188.

YBARRA, J-A. (2009), Industrial Districts in Spain, in G. Becattini, M. Belandi, de Propris L., Handbook of Industrial Districts, Cheltenham, Edgard Elgar, 512-520.

YBARRA, J-A., DOMÉNECH-SÁNCHEZ, R. (2011), Las Agrupaciones de Empresas Innovadoras y la política industrial española basada en el territorio, Economía Industrial, 380, 143-152.

YBARRA, J-A., DOMÉNECH-SÁNCHEZ, R. (2012), Innovative business groups: territory-based industrial policy in Spain, European Urban and Regional Studies, 19(2), 212-218. 